\title{
A systematic study on efficiency enhancements in phosphorescent green, red and blue microcavity organic light emitting devices
}

\author{
Chaoyu Xiang ${ }^{1}$, Wonhoe Koo ${ }^{1}$, Franky So ${ }^{1}$, Hisahiro Sasabe ${ }^{2}$ and Junji Kido ${ }^{2}$ \\ A systematic study has been conducted on microcavity organic light emitting diodes (OLEDs) based on green, red and blue \\ phosphorescent emitters to elucidate the microcavity effects for different color emitters. We found that the luminance output is \\ determined by the reflectivity of the semitransparent electrode and the photopic response of the green, red and blue emitters. \\ While the luminance enhancements of blue and red phosphorescent microcavity devices are small, a current efficiency as high as \\ $224 \mathrm{~cd} \mathrm{~A}^{-1}$ is obtained in the green phosphorescent microcavity OLEDs.
}

Light: Science \& Applications (2013) 2, e74; doi:10.1038/lsa.2013.30; published online 7 June 2013

Keywords: efficiency enhancements; microcavity; organic light emitting devices; phosphorescence; photopic luminosity

\section{INTRODUCTION}

The spectral characteristics of an organic light emitting diode (OLED) can be manipulated using a microcavity structure to produce saturated colors and enhance the color gamut, and therefore, microcavity OLEDs are widely used in active matrix OLED (AMOLED) displays today. ${ }^{1-3}$ In addition, it has been reported that microcavity structure can be used to enhance the light extraction of an OLED by modifying the light distribution within the device. ${ }^{4-7}$ However, closely examining the previous reports, there is a significant difference in luminance enhancements in different microcavity OLEDs. While two times enhancement in current efficiency has been observed in green microcavity OLEDs, no significant improvements were reported in blue microcavity devices. ${ }^{8-10}$ Moreover, there is a discrepancy between enhancement in current efficiency and enhancement in quantum efficiency. ${ }^{11}$ It is apparent that the difference in quantum efficiency and luminance enhancements cannot be due to the difference in photon outcoupling. It is therefore important to take into account the photopic luminosity due to the microcavity effects.

In this paper, we report on a systematic study of the microcavity effects on the light outcoupling efficiency and luminance output in red, green and blue phosphorescent OLEDs. Specifically, we investigated two important parameters in microcavity OLEDs: the reflectivity of the semitransparent electrode and the cavity length. A high reflectivity and low absorption semitransparent electrode is required in microcavity OLEDs so as to control the luminance characteristics. We demonstrated the enhancement in photon outcoupling of the microcavity devices can be greatly affected by the reflectivity of the electrodes. By tuning the cavity length, we found the change of photopic luminosity within the microcavity device leads to a difference in enhancements of current efficiency and quantum efficiency. Finally, a green microcavity OLEDs with a current efficiency of $224 \mathrm{~cd} \mathrm{~A}^{-1}$ was demonstrated by optimizing both the photon outcoupling efficiency and photopic luminosity.

\section{MATERIALS AND METHODS}

Indium tin oxide-coated distributed Bragg reflector (DBR) substrates composing of two pairs of quarter-wave stacks of alternating layers of titanium oxide $\left(\mathrm{TiO}_{2}\right)$ and silicon oxide $\left(\mathrm{SiO}_{2}\right)$ were used for OLED fabrication. The reflectance maxima of the DBR substrates for red, green and blue emitting devices were tuned to 610,550 and $474 \mathrm{~nm}$, respectively. After ultraviolet ozone treatment for $15 \mathrm{~min}$, a 25 -nm-thick poly(3,4-ethylenedioxythiophene)-polystyrenesulfonic acid (PEDOT:PSS) (AI 4803) as a hole injection layer was spin-coated onto indium tin oxide substrates and baked at $180{ }^{\circ} \mathrm{C}$ for $15 \mathrm{~min}$ in air. To fabricate the devices, the following layers were sequentially deposited by thermal evaporation: a 20- to 45-nm-thick 1,1-bis[(di-4-tolylamino)phenyl]cyclohexane (TAPC) as a hole transport layer, a 20 - to 30 -nm-thick 4,4-N,N-dicarbazole-biphenyl (CBP) doped with $7 \mathrm{wt} \%$ tris(2-phenylpyridine)iridium $\left(\operatorname{Ir}(\text { ppy })_{3}\right)$ as a green emitter, a 15 -nm-thick 3,5'-N, $N^{\prime}$-dicarbazolebenzene (mCP) doped with $15 \mathrm{wt} \%$ iridium (III)bis [2-methyldibenzo-(f,h) quinoxaline](acetylacetonate) $\left(\operatorname{Ir}(\mathrm{MDQ})_{2}(\mathrm{acac})\right)$ and a 15 -nm-thick 2,2',2" (1,3,5-benzenetriyl)tris-(1-phenyl-1H-benzimidazole) (TPBi) doped with $15 \mathrm{wt} \% \operatorname{Ir}(\mathrm{MDQ})_{2}(\mathrm{acac})$ as a red emitter, a 20 -nm-thick mCP doped with 5 wt\% iridium(III) bis[(4,6-difluorophenyl)pyridinato- $\left.N, C 2^{\prime}\right]$ picolinate (FIrpic) as a blue emitter, a 45- to 60-nm-thick tris[3-(3-pyridyl)-mesityl]borane (3TPYMB) as an electron transporting layer for the green and red emitting devices, a $45-$ to 55-nm-thick 3,5,3",5"'-tetra-3-pyridyl-[1,1';3',1"] terphenyl (В3РyPB) as

${ }^{1}$ Department of Materials Science and Engineering, University of Florida, Gainesville, FL 32611-6400, USA and ${ }^{2}$ Department of Polymer Science and Engineering, Yamagata University, Yamagata 992-8510, Japan

Correspondence: F So, Department of Materials Science and Engineering, University of Florida, Gainesville, FL 32611-6400, USA

E-mail: fso@mse.ufl.edu

Received 17 October 2012; revised 8 January 2013; accepted 12 January 2013 
an electron transporting layer for the blue emitting devices, a 1-nm-thick $\mathrm{LiF}$ layer as an electron injection layer and a 100-nm-thick aluminum as a cathode. To extract the substrate mode, a macrolens was attached to the substrate using an index matching gel. Current-luminance-voltage characteristics were measured using a Keithley Series 2400 source meter and a Keithley Series 6485 picoammeter with a calibrated Newport silicon photodiode. The luminance was calibrated using a Konica Minolta luminance meter (LS-100). The electroluminescence spectra were obtained with an Ocean Optics HR4000 spectrometer.

\section{RESULTS AND DISCUSSION}

The resonant emission enhancement $G_{e}$ along the normal direction in a microcavity structure is related to the reflectivity of the semitransparent mirror based on the following relationship: ${ }^{12}$

$$
G_{e} \propto \frac{\left(1+\sqrt{R_{2}}\right)^{2}\left(1-R_{1}\right)}{\left(1-\sqrt{R_{1} R_{2}}\right)^{2}}
$$

where $R_{1}$ and $R_{2}$ refer to the reflectivities of the front semitransparent mirror and back metal mirror, respectively. In order to enhance the light output from a microcavity, a front semitransparent electrode with high reflectivity and low absorption loss is preferred. A semitransparent metal electrode such as a thin Ag layer is mostly commonly used due to its simple fabrication steps. ${ }^{4,11,13-15}$ However, a thin Ag layer does not provide a high enough reflectivity for a microcavity device. Because of the large extinction coefficient of a metal and of the presence of surface plasmon mode at the organic/metal interface, the use of semitransparent metal electrodes is not favorable for microcavity OLEDs.

On the other hand, the use of dielectric DBR has the advantages of high reflectivity and low absorption loss. ${ }^{16}$ Here we chose $\mathrm{SiO}_{2} / \mathrm{TiO}_{2}$ DBRs for our microcavity OLED fabrication. Figure 1 shows the measured reflectivity of the DBR substrates for blue, green and red microcavity devices. The peak reflectivities of the blue, green and red DBR substrates were tuned to 474,550 and $610 \mathrm{~nm}$, respectively. Due to the dispersion of refractive indices of $\mathrm{SiO}_{2}$ and $\mathrm{TiO}_{2}$, the maximum reflectivities of the DBR substrates are different. The reflectivities are $62 \%$, $75 \%$ and $65 \%$ for blue, green and red DBR substrates respectively, corresponding to a calculated enhancement ratio of $0.581: 1.00: 0.685$ normalized by green enhancement according to Equation (1). Because of the higher reflectivity of the semitransparent electrodes, they give rise to a stronger microcavity effect with higher resonance peak intensity, it

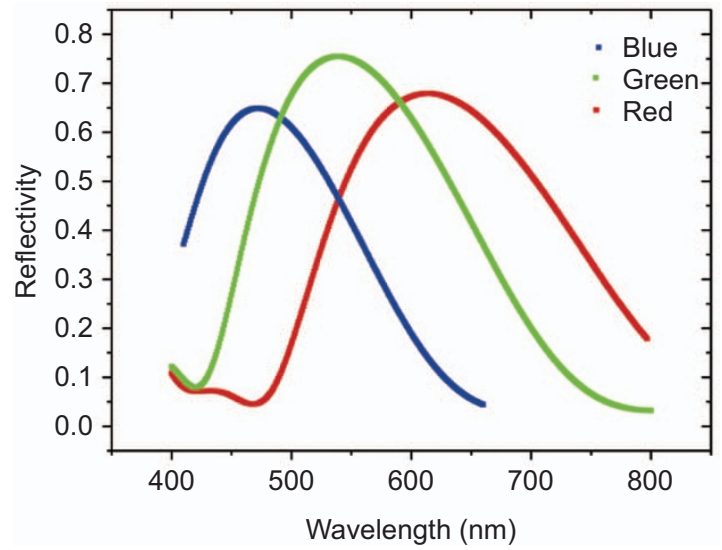

Figure 1 Reflectivity of DBR substrates for blue, green and red. DBR, distributed Bragg reflector. is expected that the green microcavity device will give a higher efficiency enhancement than the blue and red devices. We fabricated blue, green and red emitting OLEDs on DBR substrates along with devices on non-DBR substrates which were used as references. Figure $2 \mathrm{a}$ shows the structures of the optimized devices with and without microcavity structure. Figure $2 \mathrm{~b}$ shows the normalized electroluminescent (EL) spectra of the microcavity devices and the EL spectra of the reference devices driven at a constant current density. Compared to the non-cavity devices, the DBR devices show significantly enhanced luminance output along the normal direction with narrow full widths at half maximum due to the strong microcavity effects. The intensity enhancements of the blue, green and red devices at optimized wavelength are 3.5, 6.0 and 3.9, respectively. The ratio of those enhancements is $0.583: 1.00: 0.650$ for blue, green and red devices, which match the calculation from the reflectivity of each substrate.

In order to study the cavity length effect, devices with three different cavity lengths were fabricated on DBR substrates for red, green and blue OLEDs. The cavity length of these devices is based on the device structures of each emitting color as shown in Figure 2a. For the green emitting devices, we tuned the thicknesses of the TAPC and emitting layers. Device G1 has a 35-nm thick TAPC layer and a 30-nm thick CBP:Ir(ppy) ${ }_{3}$ layer, device G2 has a $30-\mathrm{nm}$ thick TAPC layer and a 25-nm thick CBP:Ir(ppy) ${ }_{3}$ layer and device G3 has a 30-nm thick TAPC layer and a 20-nm thick CBP:Ir(ppy) ${ }_{3}$ layer. Figure 3a shows the EL spectra of the devices with and without DBR driven at a current density of $0.1 \mathrm{~mA} \mathrm{~cm}^{-2}$. The non-cavity devices showed broad EL spectra with full widths at half maximum of about $80 \mathrm{~nm}$ and the intensity of shoulder peak in EL spectra is slightly reduced as the optical length decreases from device G1 to device G3 due to the weak microcavity effect. ${ }^{17}$ On the other hand, in addition to the significantly enhanced intensity along the normal direction and narrow full widths at half maximum in the microcavity devices, the peak wavelength of the DBR device decreases from $572 \mathrm{~nm}$ to $528 \mathrm{~nm}$ with decreasing thicknesses of the TAPC and emitting layers. While the main peak emission wavelength of $\operatorname{Ir}(\text { ppy })_{3}$ is around $515 \mathrm{~nm}$, the G2 DBR device exhibits a higher EL intensity than the G1 DBR device along the normal direction because the DBR substrate for green devices has the highest reflectivity at $550 \mathrm{~nm}$. We calculated the enhancement ratio due to microcavity effects by integrating the EL intensity of each device over all wavelengths and considering the ratio of the integrated intensity of the DBR device to that of the noncavity device, and the results are shown in Table 1. DBR devices G1, G2 and G3 show enhancement ratios of $60.1 \%, 101 \%$ and $70.2 \%$, respectively, indicating the enhanced outcoupling efficiency due to the microcavity effects. However, the current efficiency shows different enhancement ratios from the number of outcoupled photons. The current efficiencies for devices G1, G2 and G3 with and without DBR as shown in Figure 3b are 115,175 and $104 \mathrm{~cd} \mathrm{~A}^{-1}$, and 65,73 and $53 \mathrm{~cd} \mathrm{~A}^{-1}$ at $0.1 \mathrm{~mA} \mathrm{~cm}{ }^{-2}$, respectively, representing enhancements of $75 \%, 140 \%$ and $97 \%$ due to microcavity effects. While the G2 DBR device shows the highest quantum efficiency and current efficiency, the enhancement ratio of the current efficiency is higher than that of the quantum efficiency along the normal direction. This discrepancy in enhancement ratio is caused by the difference in luminosity. The normalized photopic luminosity $(L)$ of each spectrum is calculated according Equation (2):

$$
L=\int_{400}^{750} V(\lambda) S(\lambda) \mathrm{d} \lambda
$$


a

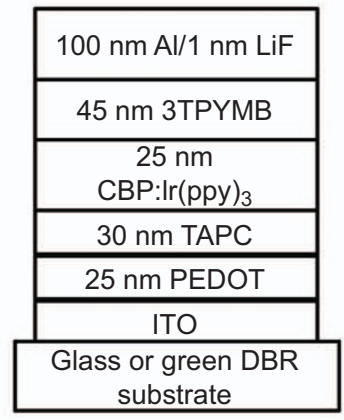

Green devices

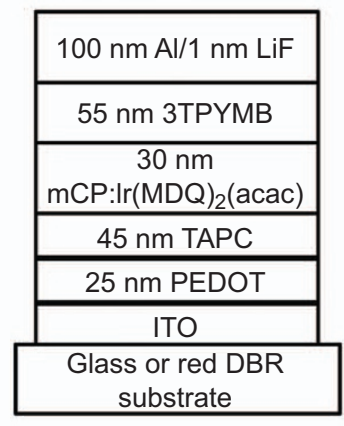

Red devices

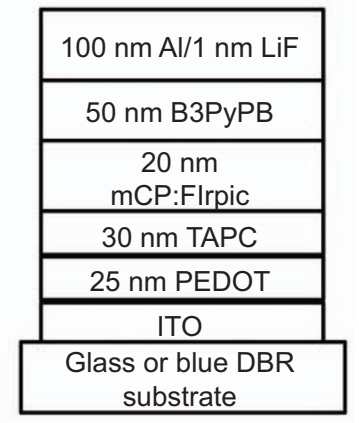

Blue devices

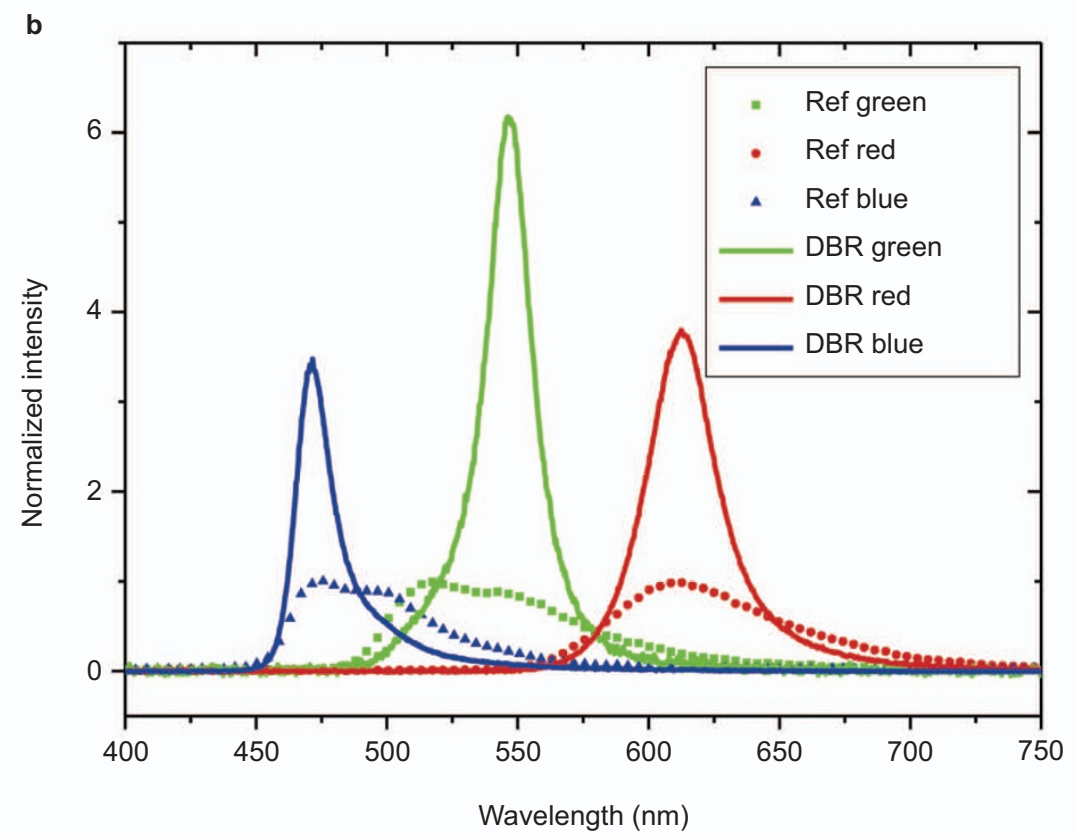

Figure 2 (a) Device structure of green, red and blue OLEDs; (b) normalized EL spectra of green, red and blue devices. Spectra were normalized to the peak intensities of their non-cavity devices with same organic structure. EL, electroluminescent; OLED, organic light emitting diode.

where $S(\lambda)$ is the normalized electroluminescent spectrum of OLEDs, $V(\lambda)$ is the standard photopic luminosity function with an unity value at $555 \mathrm{~nm}$ and $\lambda$ is wavelength. The integrating region is over all visible wavelengths. We calculated enhancement ratio of the luminosity in the G1, G2, and G3 DBR devices from their references. The results are shown in Table 1. Because the narrow EL spectra of the DBR devices are peaked at around $550 \mathrm{~nm}$ where the photopic luminosity has a highest value, all DBR devices show higher luminosities than the noncavity devices and the G2 DBR device having a maximum EL intensity at $550 \mathrm{~nm}$ represents the highest enhancement of luminosity. By considering both of enhancements from the quantum efficiency along the normal direction and the luminosity, the total enhancement ratios of $78 \%, 146 \%$ and $95 \%$ can be calculated for the G1, G2 and G3 DBR devices, respectively, and they are in agreement with the measured enhancement ratios of the current efficiencies.

We also examined the cavity length effects on red emitting phosphorescent microcavity OLEDs. Keeping the rest of the device structure the same as the red device shown in Figure 2a, we fabricated devices with different 3 TPYMB thickness: device R1 has a thickness of $52 \mathrm{~nm}$, device R2 has a thickness of $55 \mathrm{~nm}$ and device R3 has a thickness of $61 \mathrm{~nm}$. Figure 3c shows the normalized EL spectra of the devices with and without DBR driven at constant current density along the normal direction. With a significantly reduced FWMH, the emission peak decreases from $599 \mathrm{~nm}$ to $630 \mathrm{~nm}$. The R2 DBR device having a resonant wavelength at $612 \mathrm{~nm}$, corresponding to the peak reflectivity of red DBR, shows the highest enhancement of $66.8 \%$ by integrating all wavelengths, while the shorter and longer microcavity devices showed an enhancement of $46.6 \%$ and $31.4 \%$ respectively due to the mismatch of the DBR reflectivity. Figure $3 \mathrm{~d}$ shows the current efficiencies of the devices with and without DBR. At a current density of $0.1 \mathrm{~mA} \mathrm{~cm}^{-12}$, devices $\mathrm{R} 2$ with and without DBR show the highest current efficiencies of 29 and $55 \mathrm{~cd} \mathrm{~A}^{-1}$, respectively, indicating a $89 \%$ enhancement due to microcavity effects, while the R1 DBR device shows a lower current efficiency of $50 \mathrm{~cd} \mathrm{~A}^{-1}$ and the R3 DBR device has the same current efficiency as the R3 noncavity device. Compared to an enhancement of $66.6 \%$ in the EL intensity along the normal direction, a larger enhancement of $89 \%$ in current efficiency for the R2 DBR device is attributed to an increase in luminosity (Table 1). 

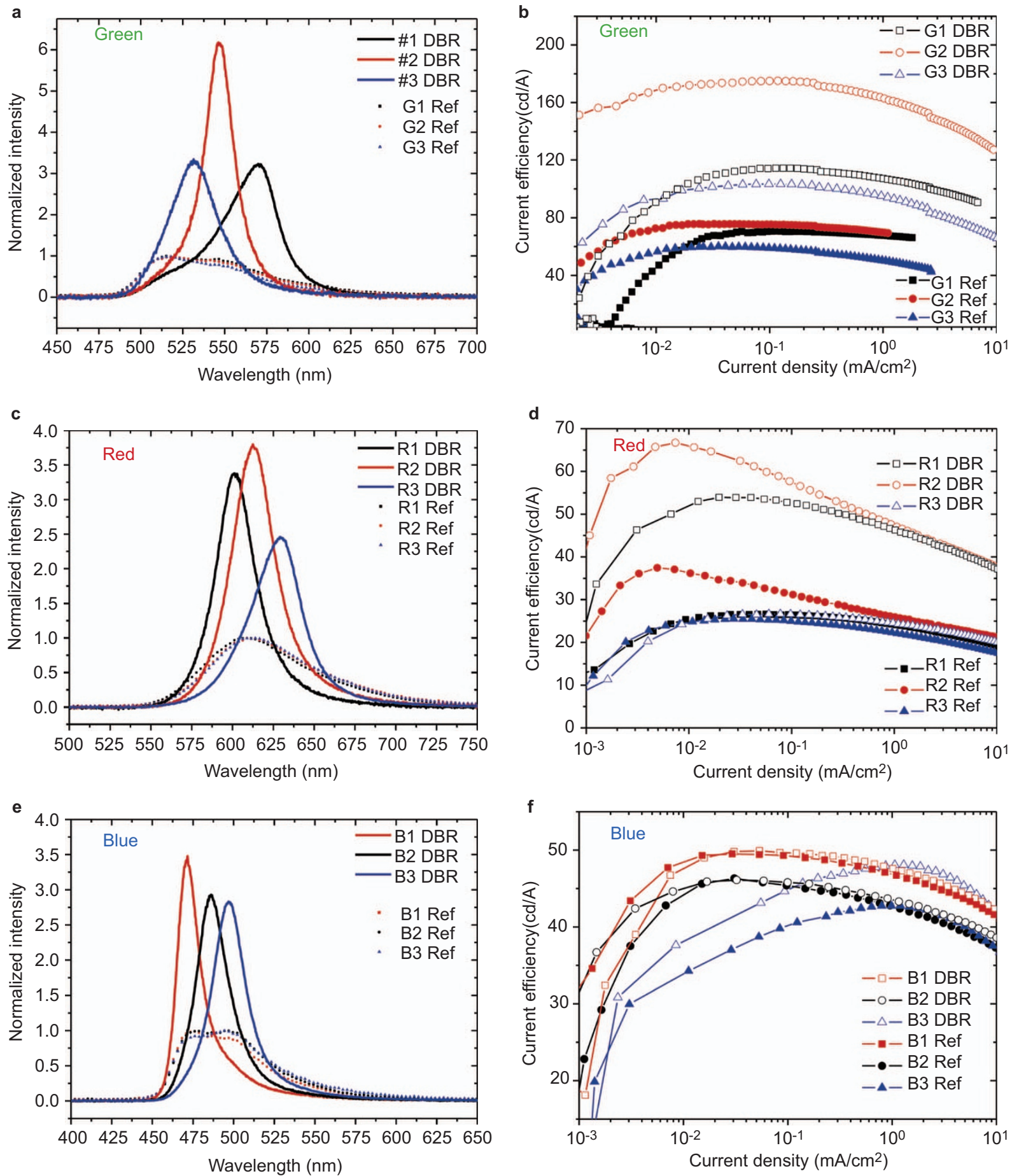

Figure 3 Normalized EL spectra of green (a), red (c) and blue (e) devices, and current efficiency of green (b), red (d) and blue (f) OLEDs. Spectra were normalized to the peak intensities of their non-cavity devices with the same organic structure. EL, electroluminescent; OLED, organic light emitting diode.

The importance of the luminosity is apparent in the R3 DBR device. While the R3 DBR device shows an enhancement of $31.4 \%$ for the integrated EL intensity along normal direction over the R3 non-cavity device, a decrease of the luminosity by $23.7 \%$ in the R3 DBR device leads to insignificant changes in current efficiency even there is an enhancement in outcoupled photons.

Finally, we investigated the microcavity effects on the blue emitting phosphorescent OLEDs. B3PyPB having high triplet energy and high mobility was used as the electron transporting layer. ${ }^{18}$ Devices B1, B2 and B3 have the thicknesses of B3РyPB layer as $45 \mathrm{~nm}, 49 \mathrm{~nm}$ and $53 \mathrm{~nm}$, respectively. Figure $3 \mathrm{e}$ shows the normalized EL spectra of the DBR devices with along the normal direction compared with the corresponding devices without DBR. With the increase of cavity length, the DBR devices showed a peak emission wavelength change from $470 \mathrm{~nm}$ to $498 \mathrm{~nm}$. While all DBR devices showed enhancements of $40.1 \%, 29.4 \%$ and $23.2 \%$ in the integrated intensity, the B1 DBR 
Table 1 Enhancement from spectra was percentage change between integrated intensities of non-cavity and microcavity having same organic structure

\begin{tabular}{|c|c|c|c|c|c|}
\hline Color & Device & $\begin{array}{l}\text { Enhancement from quantum } \\
\text { efficiency (\%) }\end{array}$ & Change of luminosity (\%) & \multicolumn{2}{|c|}{ Current efficiency enhancement (\%) } \\
\hline \multirow[t]{2}{*}{ Green } & G1 & 60.1 & 11.3 & 78 & 75 \\
\hline & G3 & 70.2 & 14.8 & 95 & 97 \\
\hline \multirow[t]{2}{*}{ Red } & R1 & 46.6 & 32.6 & 94 & 100 \\
\hline & R2 & 66.8 & 14.2 & 90 & 89 \\
\hline \multirow{2}{*}{ Blue } & B2 & 29.4 & -19.5 & 4 & 1 \\
\hline & B3 & 23.2 & -10.6 & 11 & 12 \\
\hline
\end{tabular}

device shows the highest enhancement with a resonant wavelength at $470 \mathrm{~nm}$ which corresponds to the peak reflectivity of blue DBR. However, device B3 DBR is the only device which shows an enhancement in current efficiency (Figure 3f) and devices DBR B1 and B2 showed the same current efficiencies as the non-cavity B1 and B2 devices. This is due to the significantly decreasing luminosity as the resonant wavelength of the emitting light shifts toward shorter wavelengths away from $550 \mathrm{~nm}$. According to Table 1, the luminosity in the B1, B2 and B3 DBR devices decrease by $28.7 \%, 19.5 \%$ and $10.6 \%$, respectively. Even if the B3 DBR device exhibited $12 \%$ enhancement in the current efficiency, the Commission Internationale de L'Eclairage (CIE) coordinates of its EL spectrum is $(0.06,0.51)$, indicating that the emitting color is no longer blue. Therefore, in order to achieve deeper blue from FIrpic, one has to force the microcavity peak to shorter wavelengths, which is demonstrated in device B1 DBR. Consequently, it causes a greater mismatch between the cavity resonance wavelength and the FIrpic emission spectrum, resulting in a lower enhancement in quantum efficiency. Therefore, it is difficult to enhance the efficiency of the FIrpic-based blue emitting OLED device while pushing the emission peak to shorter wavelengths to generate a deeper blue color. Figure 4 shows the CIE coordinates for the R, G and B EL spectra from the non-cavity devices and optimized microcavity devices. Because of the reduction of the EL spectral width in the microcavity devices, the CIE coordinates of the R, G and B emitters moved towards the boundary of CIE chart, from $(0.617,0.373),(0.321,0.624)$ and $(0.148,0.345)$ to $(0.636,0.350),(0.289,0.692)$ and $(0.138,0.170)$, respectively, enlarging the color gamut in the microcavity devices.

To further enhance the device efficiency, we demonstrate the microcavity effects through a highly efficient OLED device using double emitting layer (DEL). It has been demonstrated that the DEL structure can confine the emitting zone of OLEDs within the center of the emitting layer. ${ }^{19,20}$ Figure 5a shows the device structure and the energy level diagram of the DEL OLEDs. The resonant peak wavelength of the DBR device was tuned to $555 \mathrm{~nm}$ to maximize the luminance enhancement. TCTA was selected as a host due to its energy band edge offset regarding CBP. While TCTA has a hole mobility of $3 \times 10^{-4} \mathrm{~cm}^{2} \mathrm{~V}^{-1} \mathrm{~s}^{-1}$ and a very low electron mobility of $10^{-8} \mathrm{~cm}^{2} \mathrm{~V}^{-1} \mathrm{~s}^{-1}$, CBP has high electron and hole mobilities of $3 \times 10^{-4}$ and $2 \times 10^{-3} \mathrm{~cm}^{2} \mathrm{~V}^{-1} \mathrm{~s}^{-1},{ }^{21}$ respectively. As a result, exciton generation zone is confined at the TCTA/CBP interface, preventing the quenching of excitons at the interface between the emitting layer and the electron transport layer. Additionally, the DEL structure also facilities carrier injection into the emitting layer due to the low lying highest occupied molecular orbitals of TCTA. Figure 5b shows the current density-voltage characteristics of the single and double emitting layer devices with and without DBR. It is apparent that DEL devices have an order of magnitude higher in current density than that of the single emitting devices. Figure $5 \mathrm{c}$ shows the current efficiency of the DEL OLEDs with and without DBR. The devices with and without DBR show the maximum current efficiencies of 93 and $224 \mathrm{~cd} \mathrm{~A}^{-1}$, respectively, which corresponding to an enhancement of external quantum efficiency from $21 \%$ to $27 \%$. While the enhancement of $140 \%$ in the DEL OLEDs with DBR was the same as that in the \#2 DBR device with single emitting layer, the current efficiency increased to $224 \mathrm{~cd} \mathrm{~A}^{-1}$ which, to our knowledge, is the highest current efficiency for single emitting unit devices without light extraction ever reported. ${ }^{7,8}$ Figure 5d shows the angle dependence of emitting light for the noncavity device and the DBR device with and without macrolens. Both of the DBR devices with and without the macrolens showed strong forward emission distribution and with macrolens another $89 \%$ enhancement was achieved by integrating intensity at all angles. Thus, the total enhancement by a factor of $2.4(=1.28 \times 1.89)$, promising an external quantum efficiency of $50 \%$, can be achieved by the microcavity and macrolens in the DEL device with DBR. Figure 5e shows the angle dependence of peak wavelengths for non-cavity device and DBR devices with and without macrolens. Both DBR microcavity devices show blue shift with the increasing of viewing angle, while the peak wavelengths of non-cavity device stay the same.

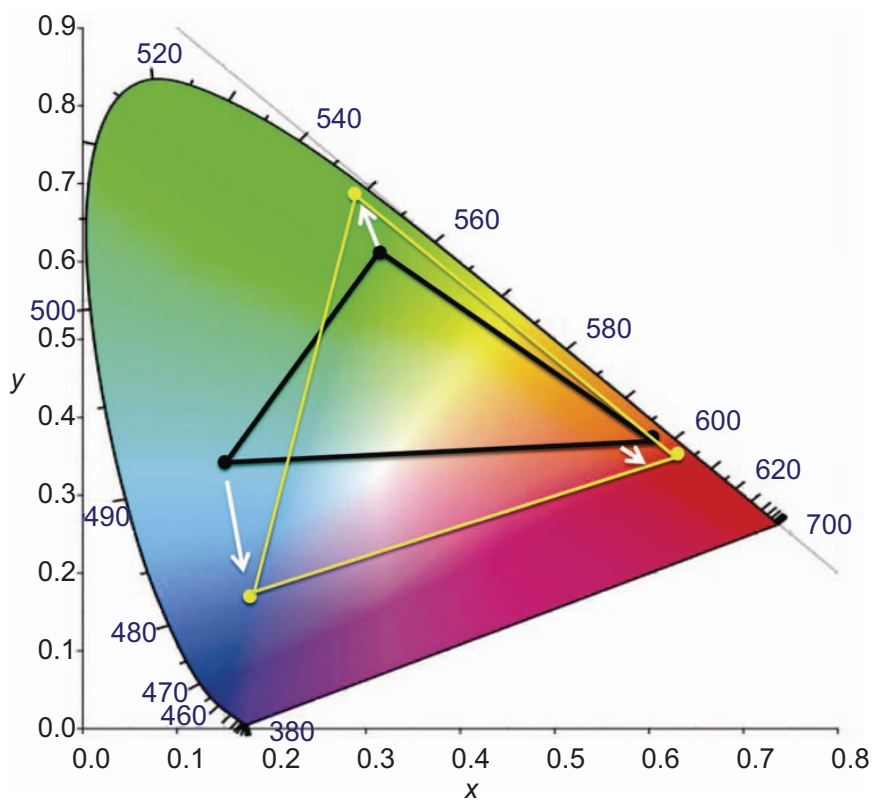

Figure 4 The Commission Internationale de L'Eclairage coordinates (1932) of non-cavity devices (black) and optimized microcavity devices (yellow) EL spectra. $\mathrm{EL}$, electroluminescent. 
a
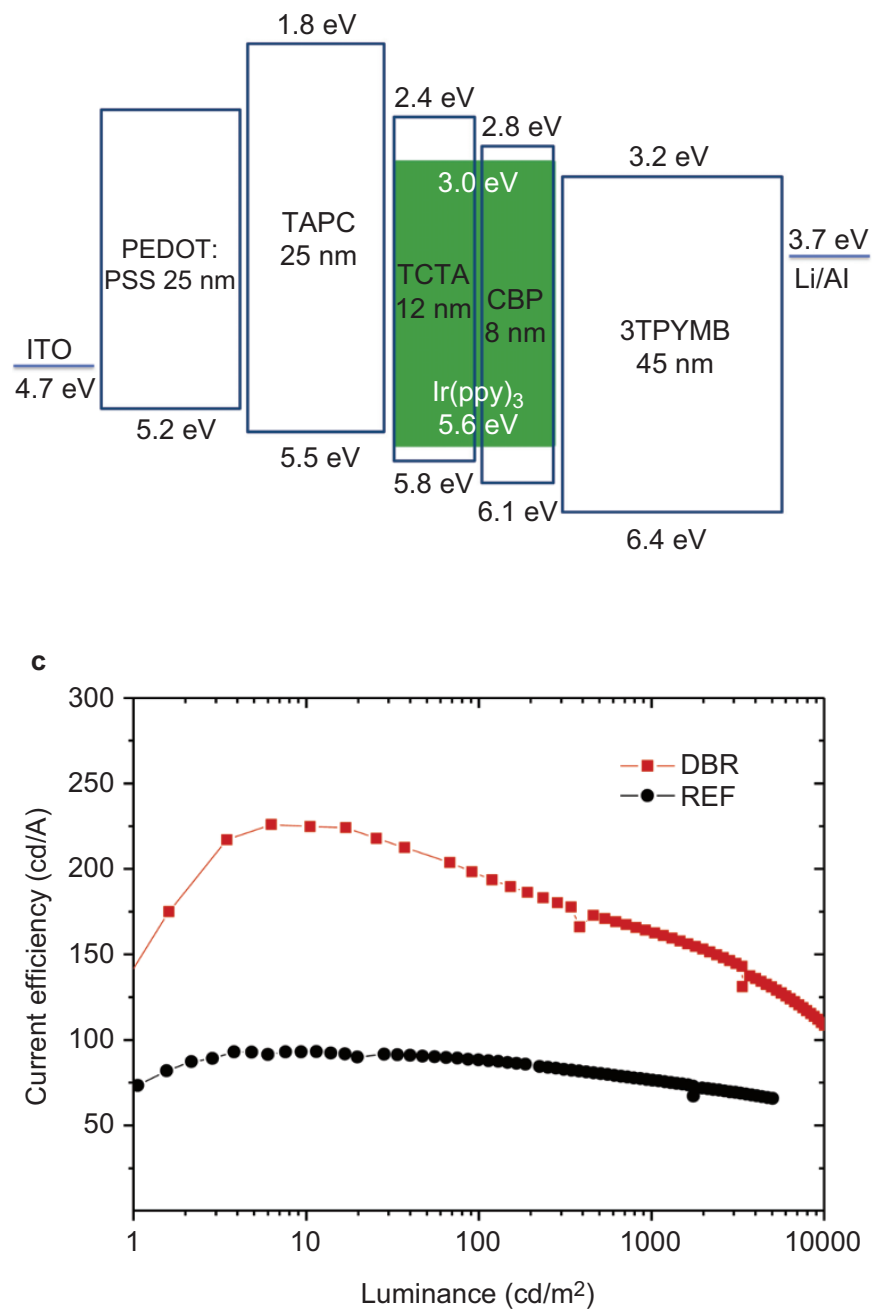

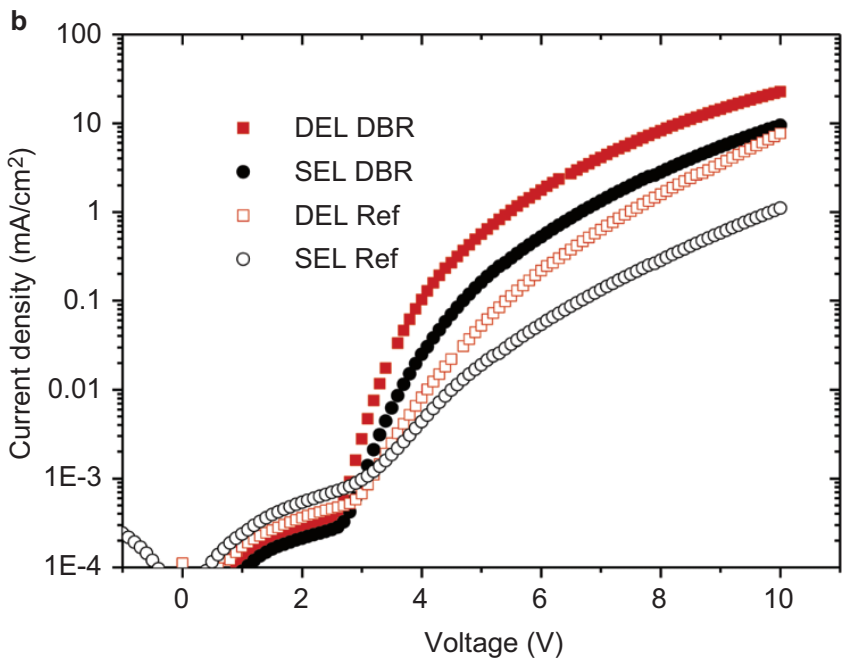

d

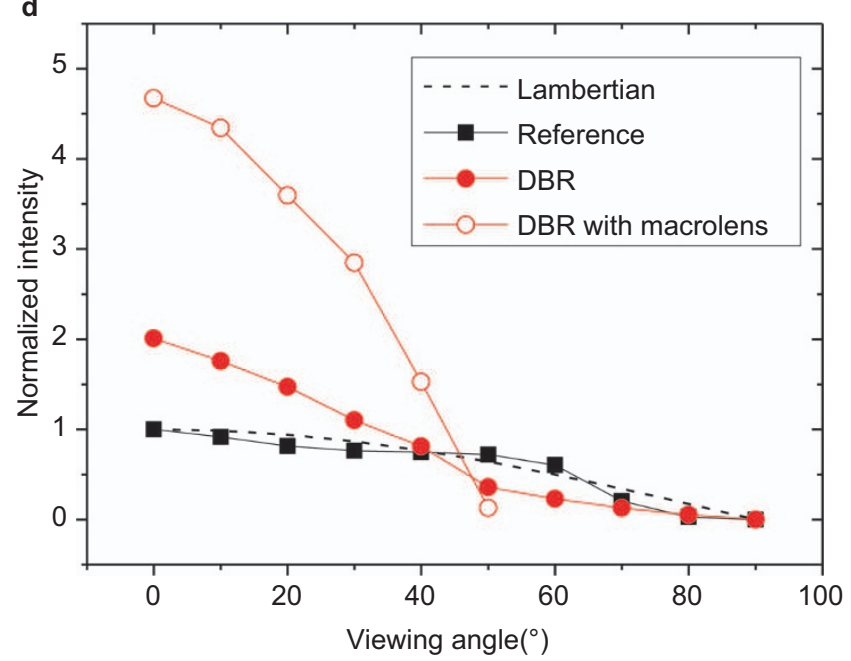

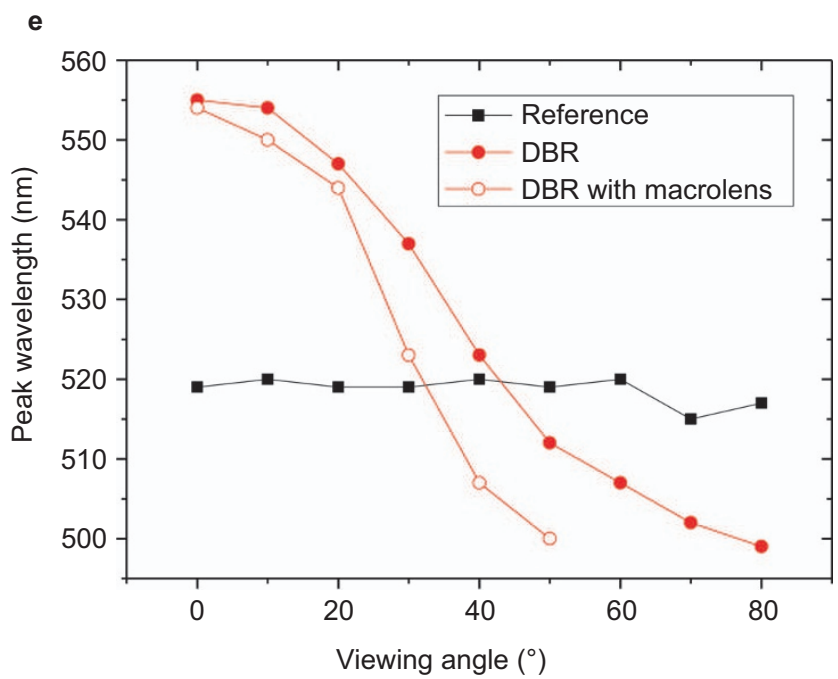

Figure 5 (a) Device structure of double emitting layer OLED; (b) current density vs. voltage of DEL and SEL devices; (c) current efficiency with and without microcavity; (d) angle dependence of integrated intensity; (e) angle dependence of peak wavelength. OLED, organic light emitting diode.

\section{CONCLUSIONS}

In summary, through a systematic study, we investigated the effects of the electrode reflectivity and cavity length on the performance of microcavity phosphorescent green, red and blue emitting OLEDs.
We found the luminance output is a strong function of the reflectivity of the electrodes as well as the EL spectral luminosity in microcavity OLEDs. The resulting enhancement in current efficiency is a combined effect of luminescence efficiency and luminosity. By optimizing 
both factors, a high current efficiency of $224 \mathrm{~cd} \mathrm{~A}^{-1}$ was demonstrated in a double-emitting-layer green microcavity OLED.

\section{ACKNOWLEDGMENTS}

We would like to acknowledge the funding support from NanoHoldings.

1 Geffroy B, Le Roy P, Prat C. Organic light-emitting diode (OLED) technology: materials, devices and display technologies. Polym Int 2006; 55: 572-582.

2 Zhou LS, Wanga A, Wu SC, Sun J, Park S et al. All-organic active matrix flexible display. Appl Phys Lett 2006; 88: 083502-083505.

3 Yang CJ, Lin CL, Wu CC, Yeh YH, Cheng CC et al. High-contrast top-emitting organic lightemitting devices for active-matrix displays. Appl Phys Lett 2005; 87: 143507-143510.

4 Peng HJ, Sun JX, Zhu XL, Yu XM, Wong M et al. High-efficiency microcavity topemitting organic light-emitting diodes using silver anode. App/ Phys Lett 2006; 88 073517-073520.

$5 \mathrm{Kim}$ HK, Cho SH, Oh JR, Lee YH, Lee JH et al. Deep blue, efficient, moderate microcavity organic light-emitting diodes. Org Electron 2010; 11: 137-145.

6 Qiu XJ, Tan XW, Wang Z, Liu GY, Xiong ZH. Tunable, narrow, and enhanced electroluminescent emission from porous-silicon-reflector-based organic microcavities. J Appl Phys 2006; 100: 074503-074509.

7 Wang ZB, Helander MG, Qiu J, Puzzo DP, Greiner MT et al, Unlocking the full potentia of organic light-emitting diodes on flexible plastic. Nat Photon 2011; 5: 753-757.

8 Cho TY, Lin CL, Wu CC. Microcavity two-unit tandem organic light-emitting devices having a high efficiency. Appl Phys Lett 2006; 88: 111106-111109.

9 Lee J, Chopra N, Bera D, Maslov S, Eom SH et al. Down-conversion white organic lightemitting diodes using microcavity structure. Adv Energy Mater 2011; 1: 174-178.

10 Lin $\mathrm{CL}$, Lin HW, Wu CC. Examining microcavity organic light-emitting devices having two metal mirrors. App/ Phys Lett 2005; 87: 021101-021104.

11 Meerheim R, Nitsche R, Leo K. High-efficiency monochrome organic light emitting diodes employing enhanced microcavities. App/ Phys Lett 2008; 93: 043310 043313.
12 Dodabalapur A, Rothberg LJ, Jordan RH, Miller TM, Slusher RE et al. Physics and applications of organic microcavity light emitting diodes. J Appl Phys 1996; 80: 6954-6964.

$13 \mathrm{Ho} \mathrm{MH}$, Wu CY, Chen TM, Chen $\mathrm{CH}$. Efficient and color-saturated inverted bottomemitting organic light-emitting devices with a semi-transparent metal-assisted electron injection layer. J Lumin 2011; 131: 78-82.

14 Li YQ, Tang JX, Xie ZY, Hung LS, Lau SS. An efficient organic light-emitting diode with silver electrodes. Chem Phys Lett 2004; 386: 128-131.

15 Zhang XW, Li J, Zhang L, Jiang XY, Haq KU et al. Top-emitting organic light-emitting device with high efficiency and low voltage using a silver-silver microcavity. Thin Solid Films 2010; 518: 1756-1759.

16 Neyts K, de Visschere P, Fork DK, Anderson GB. Semitransparent metal or distributed Bragg reflector for wide-viewing-angle organic light-emitting-diode microcavities. J Opt Soc Am B 2000; 17: 114-119.

17 Bulovic V, Khalfin VB, Gu G, Burrows PE, Garbuzov DZ et al. Weak microcavity effects in organic light-emitting devices. Phys Rev B 1998; 58: 3730-3740.

18 Sasabe H, Gonmori E, Chiba T, Li YJ, Tanaka D et al. Wide-energy-gap electrontransport materials containing 3,5-dipyridylphenyl moieties for an ultra high efficiency blue organic light-emitting device. Chem Mater 2008; 20: 5951-5953.

19 He GF, Pfeiffer M, Leo K, Hofmann M, Birnstock J et al. High-efficiency and lowvoltage $\mathrm{p}-\mathrm{i}-\mathrm{n}$ electrophosphorescent organic light-emitting diodes with doubleemission layers. Appl Phys Lett 2004; 85: 3911-3913.

20 Su SJ, Gonmori E, Sasabe H, Kido J. Highly efficient organic blue-and white-lightemitting devices having a carrier- and exciton-confining structure for reduced efficiency roll-off. Adv Mater 2008; 20: 4189-4194.

21 Kang JW, Lee SH, Park HD, Jeong WI, Yoo KM et al. Low roll-off of efficiency at high current density in phosphorescent organic light emitting diodes. Appl Phys Lett 2007; 90: 223508-223511.

(c) This work is licensed under a Creative Commons Attribution-NonCommercial-NoDerivative Works 3.0 Unported License. To view a copy of this license, visit http:// creativecommons.org/licenses/by-nc-nd/3.0 